\title{
Random amplified polymorphic DNA profiles as a tool for the characterization of Brazilian keratitis isolates of the genus Acanthamoeba
}

\author{
J.M.P. Alves ${ }^{1}$, C.X. Gusmão ${ }^{1}$, \\ M.M.G. Teixeira ${ }^{1}$, \\ D. Freitas ${ }^{2}$, A.S. Foronda ${ }^{1}$ \\ and H.T. Affonso ${ }^{1}$
}

\author{
1Departamento de Parasitologia, Instituto de Ciências Biomédicas, \\ Universidade de São Paulo, São Paulo, SP, Brasil \\ 2Departamento de O ftalmologia, Escola Paulista de Medicina, \\ Universidade Federal de São Paulo, São Paulo, SP, Brasil
}

\begin{abstract}
Correspondence
H.T. Affonso

Av. Prof. Lineu Prestes, 1374

05508-900 São Paulo, SP

Brasil

Fax: + 55-11-818-7417

E-mail: mhtaffon@ biomed.icb2.usp.br

Research supported by FAPESP and CNPq. J.M.P. Alves and C.X. Gusmão are recipients of FAPESP fellowships.
\end{abstract}

Received December 17, 1998 Accepted November 4, 1999

\begin{abstract}
The genus Acanthamoeba comprises free-living amebae identified as opportunistic pathogens of humans and other animal species. Morphological, biochemical and molecular approaches have shown wide genetic diversity within the genus. In an attempt to determine the genetic relatedness among isolates of Acanthamoeba we analyzed randomly amplified polymorphic DNA (RAPD) profiles of 11 Brazilian isolates from cases of human keratitis and 8 American type culture collection (ATCC) reference strains. We found that ATCC strains belonging to the same species present polymorphic RAPD profiles whereas strains of different species show very similar profiles. Although most Brazilian isolates could not be assigned with certainty to any of the reference species, they could be clustered according to pattern similarities. The results show that RAPD analysis is a useful tool for the rapid characterization of new isolates and the assessment of genetic relatedness of Acanthamoeba spp. A comparison between RAPD analyses and morphological characteristics of cyst stages is also discussed.
\end{abstract}

\section{Introduction}

Free-living amebae of the genus Acanthamoeba are opportunistic pathogens of humans and other animal species and have been isolated from a wide variety of natural and man-made environments $(1,2)$. In humans, some species have been implicated in different pathologies, including granulomatous amebic encephalitis (GAE), which occurs in immunologically depressed individu-
Key words

- Acanthamoeba

- Keratitis

- RAPD typing

- Genetic variation 
three distinct morphological groups (I, II and III) based primarily on endo- and ectocyst features (5). However, since the shape of cyst walls can be altered by growth conditions (6), various studies have shown that division of Acanthamoeba sp isolates into different groups and/or species is often inconsistent with the classical group designations mentioned above (7-9). Morphological parameters alone are thus not suitable for an accurate identification at the species level.

Among the molecular approaches that have been employed to discriminate species within the genus are isoenzyme patterns $(8,10)$, restriction analysis of mitochondrial DNA $(7,11)$, whole-cell DNA (12) or ribosomal DNA small subunit (SSU rDNA) (13). Based on SSU rDNA sequences, at least 12 different sequence types (T1-T12) were detected (14). Despite the demonstration of a high genetic variability within the genus, and nonetheless contributing to the classification of Acanthamoeba sp, most of these methods require large amounts of DNA, relatively complex procedures and even sequencing of the target DNA. On the other hand, the randomly amplified polymorphic DNA (RAPD) method does not require large amounts of DNA, time-consuming procedures or any a priori knowledge about the target sequences $(15,16)$. This method has been extensively used to evaluate genetic differences between related organisms (17-20).

In the present study we investigated by RAPD analysis the genetic relatedness among 19 Acanthamoeba spp, including 8 reference species from the American type culture collection (ATCC) and 11 not yet characterized Brazilian keratitis isolates. The results were used to construct data matrices and phenograms. RAPD profiles showed highly polymorphic patterns among most of the isolates studied but revealed some groups of more related organisms. The results of RAPD analysis were compared with those of morphological and morphometric analysis of cysts from the different isolates.

\section{Material and Methods}

\section{O rganisms, growth conditions and DNA extraction}

The origin and characteristics of the organisms used are listed in Table 1. For cloning purposes, isolates were grown on $2 \%$ soy extract agar plates previously seeded with heat-killed, plasmidless Escherichia coli. Two to 5 trophozoites were picked from each isolate and grown on fresh soy agar plates. After growing and encystment, one cyst of each isolate was cultured, unless otherwise specified. For large axenic cultures, cysts or trophozoites were inoculated in Neff medium (21) containing ampicillin $(100 \mu \mathrm{g} / \mathrm{ml})$ and/or gentamicin $(40 \mu \mathrm{g} / \mathrm{ml})$ and incubated at $28^{\circ} \mathrm{C}$ with shaking. DNA was purified from late-log-phase cultured cells by SDS lysis, phenol-chloroform extraction, and isopropanol precipitation, basically by the method of Kilvington et al. (22).

\section{Determination of cyst features}

To determine their size and structural features, a total of 50 cysts of each corneal scraping isolate and ATCC reference strains were photographed under the phase microscope and the main diameters were measured. The images were scanned and digitally processed to enhance quality. The average measurements of each isolate are presented in Table 1.

\section{RAPD reactions and product analyses}

A total of 35 decameric oligonucleotides of arbitrary sequence were used in a previous screening of some Acanthamoeba spp isolates. According to the number and intensity of the resulting bands, the reproducibility and discriminating potential of the amplified products, three oligonucleotides were selected for further analyses: 606 (5'CGGTCGGCCA-3'), 688 (5'-GCAGGAGC 
GT-3') and 694 (5'-GGTTTGGAGG-3'). Several of the remaining oligonucleotidederived patterns, while still useful to confirm the results of genetic similarity estimates, were not used in the computer analyses.

The DNA amplification reactions were performed in $50 \mu \mathrm{l}$ volumes containing 100 ng of template DNA, $250 \mu \mathrm{M}$ of dNTPs, 100 pmol of a single primer, 2 units of Taq DNA polymerase (Gibco BRL, Gaithersburg, MD, USA), and $1.5 \mathrm{mM} \mathrm{MgCl}_{2}$, in a Progene thermocycler (Techne, Cambridge, UK). The cycling conditions used were: initial denaturing phase of $94^{\circ} \mathrm{C}$ for $3 \mathrm{~min}$ and 35 repetitions at $94^{\circ} \mathrm{C}$ for $1 \mathrm{~min}, 37^{\circ} \mathrm{C}$ for $2 \mathrm{~min}$ and $72^{\circ} \mathrm{C}$ for $2 \mathrm{~min}$. The primer extension phase was prolonged for $10 \mathrm{~min}$ at $72^{\circ} \mathrm{C}$ in the last cycle. Amplification products were fractionated by $2 \%$ agarose gel electrophoresis, stained with ethidium bromide and photographed under ultraviolet light with an Eagle Eye II (Stratagene, La Jolla, CA, USA) image processor. The molecular marker used for fragment sizing was a 100-bp DNA ladder (Pharmacia Biotech, Uppsala, Sweden). For data analysis, amplification products obtained with each primer from each Acanthamoeba spp isolate were fractionated in the same gel.

\section{Computer-assisted analysis}

The digitized gel images were analyzed with the use of the RFLPscan Plus software (version 3.0, Scanalytics CSP Inc., Billerica, MA, USA) in order to size and compare the DNA fragments amplified from the various strains. The discrete character matrix (absence or presence of bands) was analyzed by the RAPDistance software version 1.03 (23) for the calculation of genetic distances between all compared isolates, using the Jaccard index $(A / A+B+C$, where $A$ is the number of shared bands among two organisms $\mathrm{x}$ and $\mathrm{y}$, and $B$ and $C$ are the number of bands present only in organism $\mathrm{x}$ or $\mathrm{y}$, respectively). To graphically represent the inferred groups, a phenogram was constructed by the UPGMA method using the PHYLIP 3.5 Neighbor program (24). In order to assess the robustness of the branching, a hundred bootstrap replicates were constructed and only branches presenting bootstrap values of at least 50 were considered as supported clusters.

\section{Results and Discussion}

A total of 11 Brazilian keratitis isolates and 8 ATCC reference strains, the latter belonging to 4 different species representative of the three known morphological groups of Acanthamoeba (Table 1), were characterized based on cyst morphology and RAPD analysis.

The classification of the isolates within the genus Acanthamoeba was based on morphological criteria (5). Figure 1 shows the highly characteristic double-walled morphology of cyst stages of Brazilian keratitis isolates and some representative ATCC refer-

Table 1 - Characteristics of Acanthamoeba isolates.

ATCC: American type culture collection; A. poly.: Acanthamoeba polyphaga; A. cast.: A. castellanii; HAK: human Acanthamoeba keratitis; CLW: contact lenses wearer; NCLW: noncontact lenses wearer; HCC: human choriocarcinoma cells; U/E: USP/EPM; $\mathrm{U} / \mathrm{HC}$; USP/HC; aaverage measurements of 50 cysts; btwo morphologically distinct clones isolated from the same comeal sample; cisolated from the right $(R)$ and left $(L)$ eye of the same patient.

\begin{tabular}{lllc}
\hline Isolate & ATCC & Source & Cyst diameter $(\mu \mathrm{m} \pm \mathrm{SD})^{\mathrm{a}}$ \\
\hline A. astronyxis & 30901 & water & $19.7 \pm 1.6$ \\
A. poly. \#1 & 30873 & HAK & $16.4 \pm 1.5$ \\
A. poly. \#2 & 30461 & HAK & $16.6 \pm 2.2$ \\
A. poly. \#3 & 30871 & water & $16.3 \pm 2.7$ \\
A. poly. \#4 & 30872 & water & $16.4 \pm 1.5$ \\
A. cast. \#1 & 30011 & yeast culture & $17.4 \pm 1.6$ \\
A. cast. \#2 & 30868 & HAK & $15.4 \pm 1.6$ \\
A. royreba & 30884 & HCC & $17.2 \pm 2.4$ \\
U/E 1 & & HAK-CLW & $17.0 \pm 2.2$ \\
U/E 2 & & HAK-CLW & $14.9 \pm 1.4$ \\
U/E 3 & & HAK-CLW & $16.7 \pm 1.8$ \\
U/E 4 & & HAK-NCLW & $18.3 \pm 1.6$ \\
U/E 5 & & HAK-NCLW & $14.2 \pm 1.8$ \\
U/E 6 & & HAK-CLW & $14.8 \pm 1.9$ \\
U/E 7.1 & & HAK-CLW & $14.0 \pm 1.5$ \\
U/E 7.2 & & & $18.8 \pm 2.0$ \\
U/E 8RC & & HAK-CLW & $16.5 \pm 2.3$ \\
U/E 8LC & & & $18.8 \pm 1.4$ \\
U/HC1 & & HAK-CLW & $10.8 \pm 1.4$
\end{tabular}


Figure 1 - Representative cysts of Acanthamoeba isolates photographed by phase microscopy and digitally processed. A, ATCC reference isolates - $1: A$. astronyxis; 2 : A. cast. \#1; 3: A. poly. \#3; 4: A. cast. \#2; 5: A. royreba, and 6: A. poly. \#4. B, Brazilian keratitis isolates $-1: \mathrm{U} /$ HC 1; 2: U/E 1; 3: U/E 3; 4: U/E 4; 5: U/E 5; 6: U/E 6; 7: U/7.1; 8: U/E 7.2; 9: U/E 8R; 10: U/E 8L, and 11: U/E 2. Bar $=12.0 \mu \mathrm{m}$. For abbreviations, see legend to Table 1.
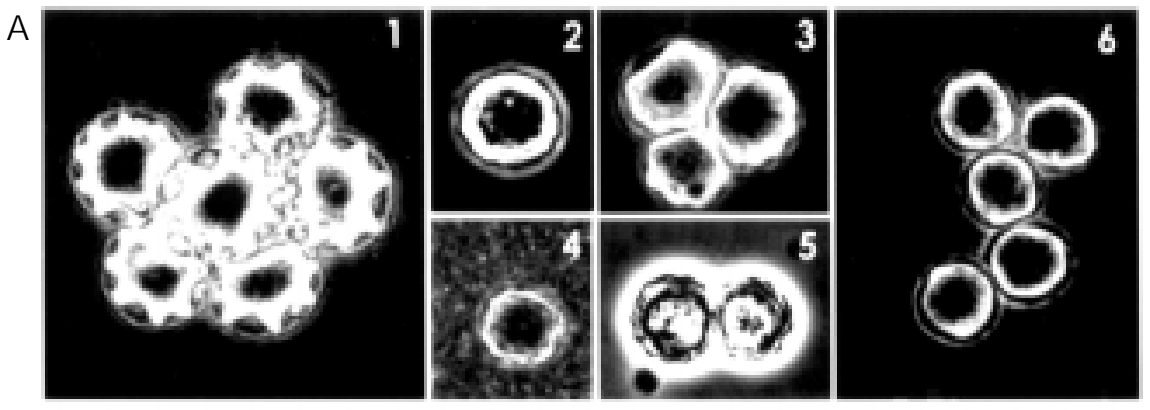

B
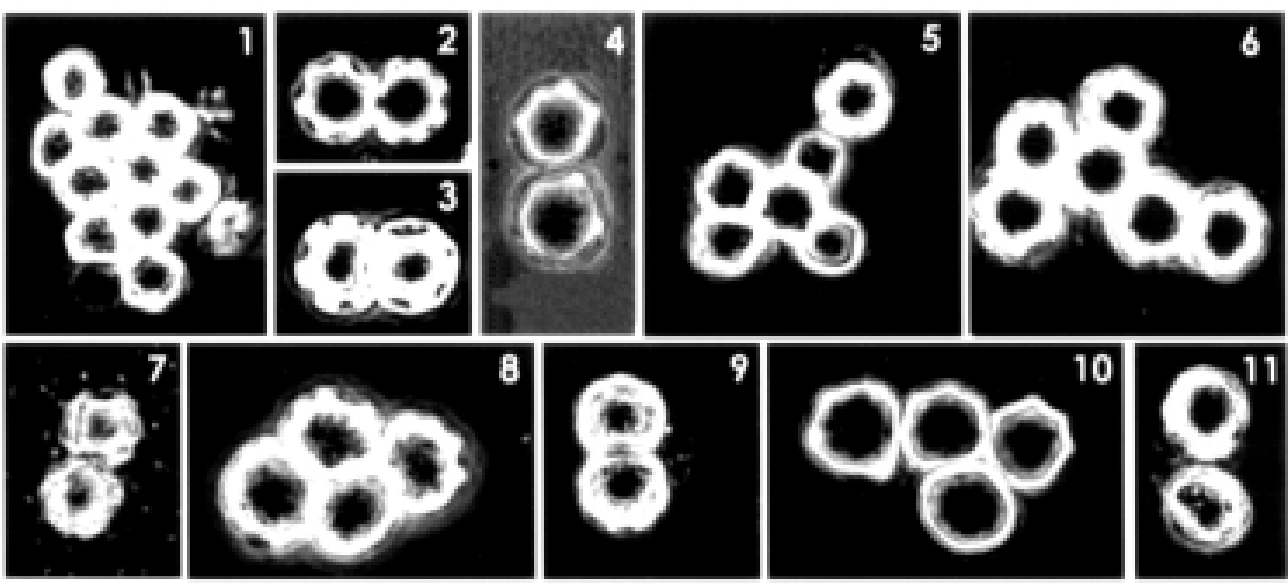

ence strains. Except for $A$. astronyxis, which presents large cysts with a smooth ectocyst and a stellate endocyst typical of group I, and A. royreba, typical of group III, the shape features of cysts were closely similar among the different isolates (Figure 1). On the other hand, average cyst sizes obtained from our keratitis isolates showed wide variability, ranging from 10.8 to $18.8 \mu \mathrm{m}$ (Table 1). In spite of this high variability, the morphological criteria allowed us to classify keratitis isolates in the genus Acanthamoeba, although they proved insufficient to distinguish among most of them. Based on these criteria, they could be included in morphological group II which comprises the majority of species (25).

To determine the genetic relatedness of the isolates, DNA fingerprints were determined by RAPD analysis. Each reaction was repeated at least three times, and 100 stable bands were considered and computed in the matrices. In most cases, RAPD profiles al- lowed detection of extensive polymorphism among isolates. Similar results were obtained with the three primers and an illustrative profile is shown in Figure 2.

Results from the RAPD analyses with the three primers were combined and used to calculate a matrix of pairwise similarities (Figure 3). This matrix was used to construct a phenogram, after determination of bootstrap values for 100 replicates (Figure 4). As expected from the RAPD profiles, some genetically more related Brazilian keratitis isolates could be detected. However, as a result of the high genetic variability, no major groups could be assigned. Moreover, none of our keratitis isolates could be surely grouped with any ATCC reference strains. Although minor differences in RAPD profiles were detected, four clusters of Brazilian keratitis isolates could be identified: a) U/E 1 and 3, b) U/E 4, 5 and 6, c) U/E 7.1 and 7.2, and d) U/E 8R and 8L. Isolates U/E 2 and U/ $\mathrm{HC} 1$ could not be assigned to any cluster 


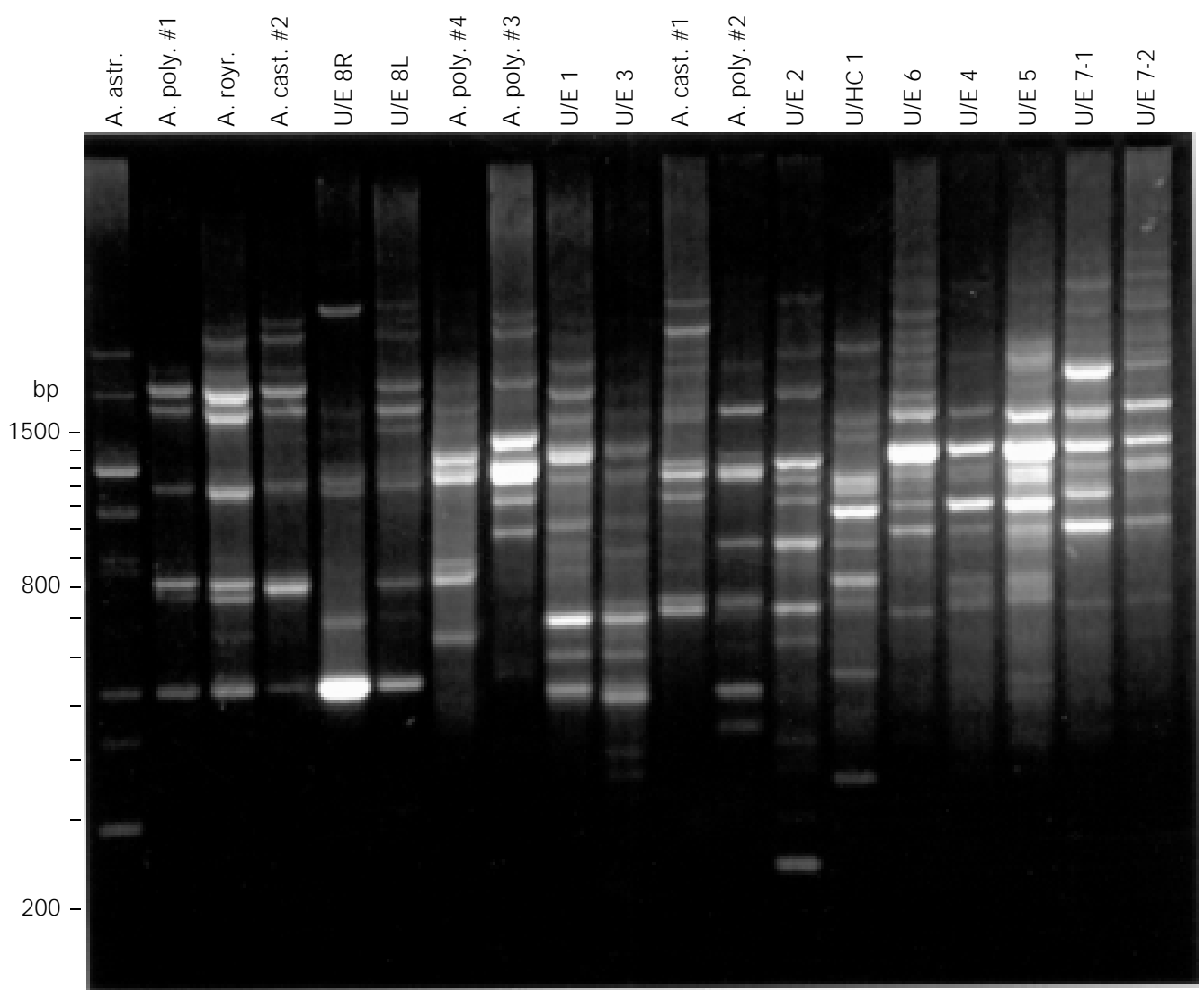

Figure 2 - RAPD profiles of 19 Acanthamoeba isolates obtained with primer 688 (5'-GCAGGA GCGT-3'). The PCR products were resolved by electrophoresis on $2 \%$ agarose gel. Designations of isolates are indicated on the top: A. astr.: A. astronyxis, A. poly.: A. polyphaga, A. cast.: A. castellanii, A. royr.: A. royreba, U/E: USP/EPM, U/HC: USP/HC. Molecular size markers (100-bp DNA ladder - Gibco BRL) are shown on the left.

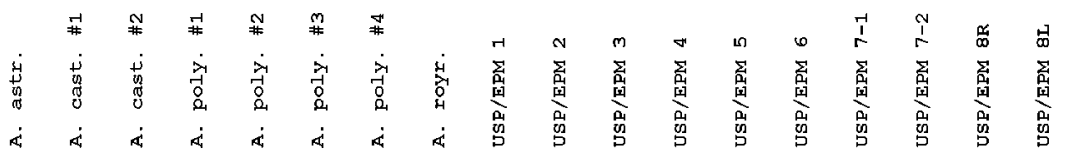

A. cast. \#1 10.0

A. cast. \#2 12.930 .4

A. poly. \#1 26.726 .945 .8

A. poly. \#2 $11.4 \quad 25.920 .018 .1$

A. poly. \#3 $6.4 \quad 16.7 \quad 11.1 \quad 13.8 \quad 13.3$

A. poly. \#4 $15.4 \quad 19.0 \quad 22.7 \quad 25.0 \quad 14.8 \quad 8.7$

A. royr.

USP/EPM 1

USP/EPM 2

USP/EPM 3

USP/EPM 4

USP/EPM 5

USP/EPM 6

USP/EPM 7-1

USP/EPM 7-2

USP/EPM $8 R$

USP/EPM 8L

USP/HC 1 $\begin{array}{lllllllllll}7.6 & 20.7 & 19.4 & 17.6 & 17.1 & 12.9 & 10.3 & 14.3 & 17.1\end{array}$

$\begin{array}{lllllllllllll}16.7 & 15.4 & 14.3 & 20.7 & 16.1 & 7.1 & 12.5 & 16.7 & 50.0 & 15.6\end{array}$

$\begin{array}{lllllllllll}13.3 & 25.1 & 19.2 & 21.4 & 25.0 & 16.0 & 30.0 & 25.9 & 20.7 & 12.5 & 19.2\end{array}$

$\begin{array}{llllllll} & \end{array}$

$\begin{array}{llllllllllllll}8.8 & 23.1 & 17.2 & 15.6 & 26.7 & 23.1 & 20.8 & 15.6 & 18.7 & 18.2 & 9.7 & 26.9 & 40.0 & 28.6\end{array}$

$\begin{array}{llllllllllllllll}10.0 & 16.7 & 15.4 & 22.2 & 13.3 & 12.0 & 19.0 & 22.2 & 25.9 & 9.4 & 11.1 & 16.0 & 14.8 & 14.3 & 18.5 & 17.9\end{array}$

$\begin{array}{llllllllllllllll} & & \end{array}$

$\begin{array}{lllllllllllllllllll}15.2 & 13.8 & 9.4 & 15.2 & 11.4 & 17.9 & 7.1 & 18.8 & 11.4 & 11.1 & 9.4 & 13.3 & 20.0 & 19.4 & 8.8 & 8.6 & 10.0 & 12.9\end{array}$
$26.7 \quad 22.2 \quad 40.0 \quad 65.2 \quad 18.2 \quad 13.825 .0$

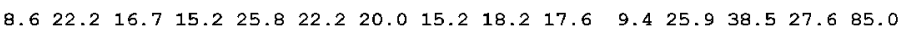

Figure 3 - Matrix showing pairwise similarities calculated for RAPD profiles obtained with primers 606, 688 and 694. For abbreviations, see legend to Figure 2 . 
Figure 4 - Unrooted UPGMA phenogram based on RAPD profiles of 19 Acanthamoeba isolates obtained with primers 606 , 688 and 694. For abbreviations, see legend to Figure 2 . The numbers at the nodes represent bootstrap values for a hundred replicates.

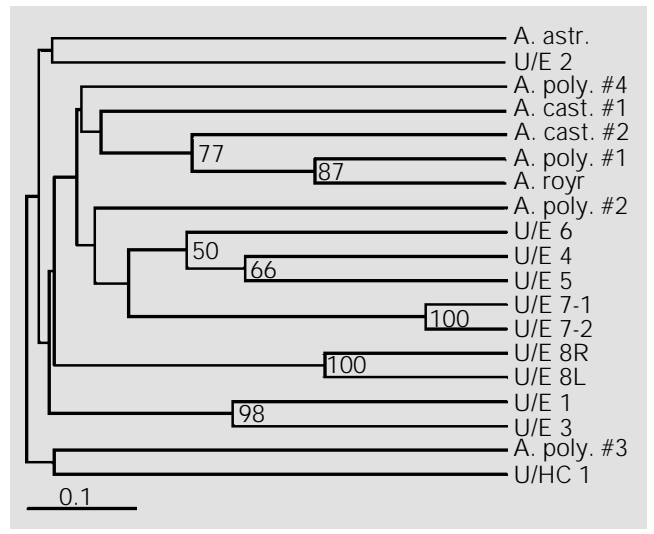

since they showed high genetic variability when compared to the others. Based on the analysis of the resulting clusters, we found both agreements and disagreements between RAPD profiles and morphological cyst features. In the clusters harboring U/E 1 and 3, and U/E 5 and 6, the isolates showed very similar cyst sizes (Table 1, Figure 1) and RAPD patterns (Figure 2), despite the fact that they were isolated from different patients. In contrast, the clusters to which $\mathrm{U} / \mathrm{E}$ 4 and 5, U/E 7.1 and 7.2, and U/E 8R and 8L were assigned showed discordance between cyst sizes and RAPD patterns. The cyst sizes of isolates U/E 4 and 5 were $18.33 \mu \mathrm{m}$ and $14.17 \mu \mathrm{m}$, respectively, whereas their RAPD profiles were very similar (Figure 2).

It is noteworthy that $\mathrm{U} / \mathrm{E} 7.1$ and 7.2, and $\mathrm{U} / \mathrm{E} 8 \mathrm{~L}$ and $\mathrm{R}$ also showed discrepancies between cyst sizes and RAPD profiles. The two clones of U/E 7, which were simultaneously isolated from the same corneal sample, showed quite different cyst sizes (Table 1, Figure 1). These observations may suggest that the patient had been infected with genetically different amebae. However, despite some detected differences, especially with primer 688 (similarity index of 0.87 ), very similar RAPD patterns were obtained (Figure 2), indicating that the two clones probably originated from the same organism. In fact, it has been reported that cyst morphology might vary depending on culture conditions, even within cloned strains $(6,26)$, possibly explaining the polymorphism of cyst sizes. Isolates U/E $8 \mathrm{R}$ and $8 \mathrm{~L}$, which were respectively obtained from the right and left eye of another patient, showed very similar cyst features (Table 1). However, the RAPD patterns with primer 688 (Figure 2) showed a lower similarity index $(0.57)$ when compared with that of the two clones of U/E 7. Analyses with other primers showed similar results (data not shown), indicating that this patient could have been infected by genetically different organisms.

A remarkable feature of the RAPD-derived phenogram is the presence of strains assigned to the same species (A. castellanii and $A$. polyphaga) distributed through different branches. On the other hand, different species - A. poly. \#1, A. cast. \#2 and A. royreba - were assigned to nearby branches. These observations do not agree with the morphological data, especially with cyst diameter. For example, the four strains designated as A. polyphaga (morphological group II) showed a narrow range of cyst sizes and A. royreba showed cyst shapes and measurements typical of morphological group III (Table 1). In contrast, $A$. astronyxis, a representative species of morphological group I, could not be clustered with any other reference species or our keratitis isolates, showing concordance between RAPD profiles and cyst features. Similar findings were also observed by other approaches $(8,14,27)$. Therefore, it is clear that species definition in the genus Acanthamoeba - especially those assigned to either A. polyphaga or A. castellanii - must be reviewed, as recently proposed (14).

Our data demonstrate the ability of RAPD analysis to assign a specific genetic fingerprint to otherwise morphologically indistinguishable Acanthamoeba isolates. Furthermore, this approach proved to be useful in the characterization of new isolates and for the assessment of genetic relatedness and proved to be a fast and informative strategy to determine relationships among different strains. A general limitation of our technique 
is its inability to properly infer phylogenetic relationships, because genetic distances calculated from RAPD could be affected by paralogy, which would make RAPD patterns inconsistent with speciation events. Even identification could be hampered by such problems, since some patterns could eventually mislead species assignment. However, our RAPD analyses are in agreement with preliminary SSU rDNA fingerprinting profile determination, which is in progress in our laboratory, indicating that this problem probably does not affect our analyses. This is nonetheless very serious and should be addressed with care in future studies.

Other approaches, such as sequencing of ribosomal DNA subunits, can be used for a more precise determination of the relationships among our strains. However, such relationships cannot be assessed if the approach used is unable to detect subtle differences between very closely related organisms, as recently found in the analysis of SSU rDNA sequences (14). Based on this last approach, at least 12 different sequence types (T1-T12) were detected, presenting dissimilarities ranging from 5.0 to $37.6 \%$ between sequence types. The detection of such divergent SSU rDNA sequences is in accordance with our data, since it also reflects the high polymorphism of these organisms. However, as stated by those authors, this strategy could not identify branching patterns within $\mathrm{T} 4$ sequence type strains, a group to which 24 of 25 worldwide-distributed keratitis isolates were assigned. Although our keratitis isolates were not characterized by that approach, they showed a high degree of polymorphism and hence were distributed through different branches by our RAPD analyses. It would thus be interesting to investigate whether these isolates present the same SSU sequence types or, conversely, if RAPD patterns of the isolates designated as T4 would be so polymorphic as to allow the identification of their branching patterns. It should be noted that three ATCC reference strains - A. poly. \#1 (30873), A. poly. \#3 (30871), and A. cast. \#1 (30011) - which were assigned to the same sequence type (T4), were also spread across different branches of the phenogram (Figure 4).

In conclusion, the RAPD technique can be useful to distinguish between closely related Acanthamoeba sp isolates and to possibly characterize additional isolates of this genus. On the other hand, more conserved sequences would still be required to determine major groups involving less related strains.

\section{Acknowledgments}

The authors thank Annamaria S. Stolf, Regina V. Milder and Silvia C. Alfieri for a critical reading of the manuscript, and Manoel A. Peres for technical assistance.

\section{References}

1. Ma P, Visvesvara GS, Martinez AJ, Theodore FH, Dagget PM \& Sawyer TK (1990). Naegleria and Acanthamoeba infections: review. Reviews of Infectious Diseases, 12: 490-513.

2. Kilvington $S \&$ White DG (1994). Acanthamoeba: biology, ecology and human disease. Reviews in Medical Microbiology, 5: $12-20$

3. Logar J \& Kraut A (1997). Acanthamoeba comeal infection in a contact lens wearer. J ournal of Infection, 35: 237-240.

4. Szénási Z, Endo T, Yagita $K \&$ Nagy E
(1998). Isolation, identification and increasing importance of "free-living" amoebae causing human disease. J ournal of Medical Microbiology, 47: 5-16.

5. Pussard R \& Pons M (1977). Morphologie de la paroi kystique et taxonomie du genre Acanthamoeba (Protozoa, Amoebida). Protistologica, 13: 557-598.

6. Stratford MP \& Griffiths AJ (1978). Variations in the properties and morphology of cysts of Acanthamoeba castellanii. J ournal of General Microbiology, 108: 33-37.

7. Costas M, Edwards SW, Lloyd D, Griffiths
AJ \& Turner G (1983). Restriction enzyme analysis of mitochondrial DNA of members of the genus Acanthamoeba as an aid in taxonomy. FEMS Microbiology Letters, 17: 231-234.

8. Daggett P, Lipscomb D, Sawyer TK \& Nerad TA (1985). A molecular approach to the phylogeny of Acanthamoeba. BioSystems, 18: 399-405.

9. Visvesvara GS (1991). Classification of Acanthamoeba. Reviews of Infectious Diseases, 13: S369-S372.

10. Costas M \& Griffiths AJ (1985). Enzyme 
composition and the taxonomy of Acanthamoeba. J ournal of Protozoology, 32: 604-607.

11. Yagita K \& Endo T (1990). Restriction enzyme analysis of mitochondrial DNA of Acanthamoeba strains in J apan. J ournal of Protozoology, 37: 570-575.

12. McLaughlin GL, Brandt FH \& Visvesvara GS (1988). Restriction fragment length polymorphisms of the DNA of selected Naegleria and Acanthamoeba amoebae. J ournal of Clinical Microbiology, 26: 16551658.

13. Kong HH \& Chung DI (1996). PCR and RFLP variation of conserved region of small subunit ribosomal DNA among Acanthamoeba isolates assigned to either A. castellanii or A. polyphaga. Korean J ournal of Parasitology, 34: 127-134.

14. Stothard DR, Schroeder-Diedrich JM, Awwad MH, Gast RJ, Ledee DR, Rodriguez-Zaragoza S, Dean CL, Fuerst PA \& Byers TJ (1998). The evolutionary history of the genus Acanthamoeba and the identification of eight new 18S rRNA gene sequence types. J ournal of Eukaryotic Microbiology, 45: 45-54.

15. Welsh J \& McClelland M (1990). Fingerprint genomes using PCR with arbitrary primers. Nucleic Acids Research, 18: 7213-7218.

16. Williams J GK, Kubelik AR, Livak KJ, Rafalski J A \& Tingey SV (1990). DNA poly- morphisms amplified by arbitrary primers are useful as genetic markers. Nucleic Acids Research, 18: 6531-6535.

17. Steindel $M$, Dias Neto $E$, Carvalho Pinto CJ, Grisard EC, Menezes CLP, Murta SMF, Simpson AJ G \& Romanha AJ (1994). Randomly amplified polymorphic DNA (RAPD) and isoenzyme analysis of Trypanosoma rangeli strains. J ournal of Eukaryotic Microbiology, 41: 261-267.

18. Pogue GP, Koul S, Lee NS, Dwier DM \& Nakhasi HL (1995). Identification of intraand interspecific Leishmania genetic polymorphisms by arbitrary primed polymerase chain reactions and use of polymorphic DNA to identify differentially regulated genes. Parasitology Research, 81: 282-290.

19. Pélandakis $M$, Kaundun SS, De J onckeheere J F \& Permin P (1997). DNA diversity among the free-living amoeba, Naegleria fowleri, detected by the random amplified polymorphic DNA method. FEMS Microbiology Letters, 151: 31-39.

20. Tibayrenc M (1998). Genetic epidemiology of parasitic protozoa and other infectious agents: the need for an integrated approach. International J ournal of Parasitology, 28: 85-104.

21. Neff RJ, Ray SA, Benton WF \& Wilborn M (1964). Induction of synchronous encystment (differentiation) in Acanthamoeba sp. In: Prescott DM (Editor), Methods in
Cell Physiology. Academic Press, New York.

22. Kilvington $S$, Beeching J \& White DG (1991). Differentiation of Acanthamoeba strains from infected corneas and the environment by using restriction endonuclease digestion of whole-cell DNA. J ournal of Clinical Microbiology, 29: 310-314.

23. Armstrong J , Gibbs A, Peakall R \& Weller $G$ (1994). RAPDistance Programs, Version 1.03 for the Analysis of Pattems of RAPD Fragments. Australian National University, Canberra.

24. Felsenstein J (1989). Phylip 3.2 Manual. University of California, Herbarium, Berkeley, CA.

25. Walker CWB (1996). Acanthamoeba: ecology, pathogenicity and laboratory detection. British J ournal of Biomedical Science, 53: 146-151.

26. J acobson LM \& Band RN (1987). Genetic heterogeneity in a natural population of Acanthamoeba polyphaga from soil, an isoenzyme analysis. J ournal of Protozoology, 34: 83-86.

27. Bogler SA, Zarley CD, Burianek LL, Fuerst PA \& Byers TJ (1983). Interstrain mitochondrial DNA polymorphism detected in Acanthamoeba by restriction endonuclease analysis. Molecular and Biochemical Parasitology, 8: 145-163. 